\title{
Auf der Suche nach der richtigen Marketinglogik
}

\author{
Literaturhinweise zusammengestellt von Alexander Schagen, MBA
}

\section{Bücher}

Belz, Ch. (2006)

Spannung Marke - Markenführung für komplexe Unternehmen, Wiesbaden.

Belz, Ch. (2009)

Marketing gegen den Strom, St. Gallen.

Belz, Ch./Reinecke, S./Reinhold, M./Schmitz, Ch./Schögel, M./Zupancic, D. (2009)

Marketing und Vertrieb in einer neuen Welt, St. Gallen.

Belz, Ch./Schögel, M./Tomczak, T. (2007) Innovation Driven Marketing - Vom Trend zur innovativen Marketinglösung, Wiesbaden.

Dannenberg, H./Zupancic, D. (2008)

Spitzenleistungen im Vertrieb - Optimierung in Vertriebs- und Kundenmanagement, Wiesbaden.

\section{Esch, F.-R. (2008)}

Strategie und Technik der Markenführung, 5. Auflage, München.

Gladwell, M. (2002)

Tipping Point: Wie kleine Dinge Großes bewirken können, 5. Auflage, München.

Godin, S. (1999)

Permission Marketing, New York.

Hagel III, J./Brown, J. S./Davison, L. (2010)

The Power of Pull. How Small Moves, Smartly Made, Can Set Big Things in Motion, New York.
Herrmann, A./Huber, F. (Hrsg.)(2009)

Produktmanagement: Grundlagen - Methoden - Beispiele, 2. Auflage, Wiesbaden.

Kim, W. C./Mauborgne, R. (2005)

Blue Ocean Strategy, Boston.

Peters, Th. J./Waterman, R. H. (2004)

Auf der Suche nach Spitzenleistungen, 10. Auflage, Frankfurt.

Prahalad, C. K./Ramaswamy, V. (2004)

The Future of Competition - Co-Creating Unique Value with Customers, Boston.

Rutschmann, M. (2005)

Kaufprozesse von Konsumenten erkennen und lenken: Mehr Marktanteil mit neuem Marketing, Heidelberg.

Sheth, J. N./Sisodia, R. S. (Hrsg.)(2006)

Does Marketing Need Reform? Fresh Perspectives on the Future, New York.

Stadelmann, M./Wolter, S./Tomczak, T./Reinecke, S. (2003)

Customer Relationship Management, Zürich.

\section{Artikel}

Belz, Ch./Huber, D./Okonek, Ch./Rutschmann, M. (2011)

Reales Kundenverhalten und reales Marketing - Analytisches Customer Relationship Management und Microkundenprozesse, in:
Belz, Ch. (Hrsg.): Innovationen im Kundendialog, Wiesbaden, S. 35-68.

Belz, Ch./Schagen, A. (2011)

Inbound Marketing fördert und nutzt die Initiative des Kunden, in: Belz, Ch. (Hrsg.) Innovationen im Kundendialog, Wiesbaden, S. 107-176.

Lusch, R. F./Vargo, S. L./Malter, A. J. (2006) Marketing as Service-Exchange: Taking a Leadership Role in Global Marketing Management, in: Organizational Dynamics, 35, 3, S. 264-278.

Maglio, P./Vargo, S. L./Caswell, N./Spohrer, J. (2009)

The Service System is the Basic Abstraction of the Service Science, in: Information Systems and E-Business Management, 7, 4, S. 395-406.

Merz, M. A./Yi He/Vargo, S. L. (2009)

The Evolving Brand Logic: A Service-dominant Logic Perspective, in: Journal of the Academy of Marketing Science, 37, 3, S. 328-344.

Vargo, S. L./Lusch, R. F. (2004)

Evolving to a New Dominant Logic for Marketing, in: Journal of Marketing, 68, 1, S. 1-17.

\section{Vargo, S. L. (2008)}

Customer Integration and Value Creation: Paradigmatic Traps and Perspectives, in: Journal of Service Research, 11, 2, S. 211-215.

\section{Fir Thren optimalen IT-Schutz + IT-Sicherheit}

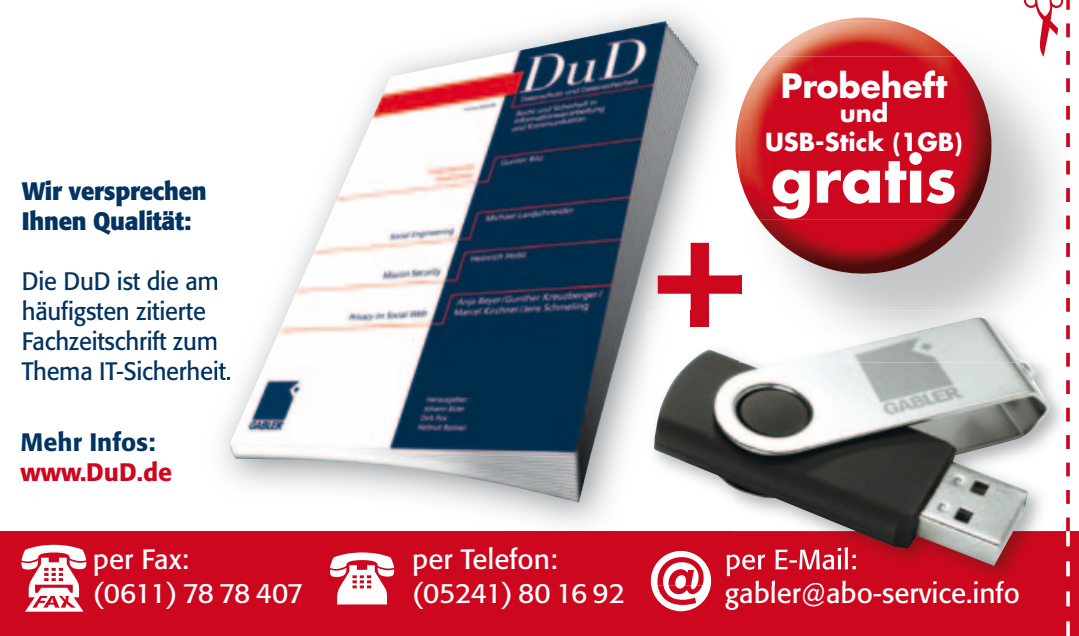

\section{Gutschein}

YI JA, ich möchte 1 Ausgabe

JA, ich möchte 1 Ausgabe
"DuD - Datenschutz und Datensicherheit" + den 1GB USB-Stick - kostenlos!

Den praktischen IGB USB-Stick schicken Sie mir mit dem Heft. Wenn mir
DuD - Datenschutz und Datensicherheit" gefällt, brauche ich nichts weiter "DuD - Datenschutz und Datensicherheit" geällt, brauche ich nichts weiter
zu tun. Ich erhalte dann „DuD" für mindestens 1 Jahr 12 Hefte) zum derzeitigen Vorzugs-Preis von nur 288,00 Euro zuzüglich Versand. Gefällt
mir "DuD" nicht, melde ich mich innerhalb von 10 Tagen nach Erhalt des mir "DuD” nicht, melde ich mich innerhalb von 10 Tagen nach Erhalt des kostenlosen Probeeftes schriftlich. (Ein Angebot der GWV Fachverlage
GmbH, GF: Dr. R. Birkelbach, A.F. Schirmacher, AG Wiesbaden HRB 9754)

Wir verspreche Ihnen Qualitat: Fachzeitschrift zum

Mehr Infos: www.DuD.de

\section{Firma,}

Name, Vorname

Straße, Nr. 